\title{
Erratum to: Life cycle assessment of a waste lubricant oil management system
}

\author{
Ana Pires • Graça Martinho
}

Published online: 12 February 2014

(C) Springer-Verlag Berlin Heidelberg 2014

Erratum to: The International Journal of Life Cycle Assessment

DOI 10.1007/s11367-012-0455-2

The original version of this article unfortunately contained a mistake.

Table 1: the legend should be: Input and output quantities of the mild reprocessing methods
Table 2: the legend should be: Input and output quantities of the recovery technologies

The values in Table 2 do not reflect all flows inside of the complex process Electric energy from WLO recycling and energy recover. The correct values are shown in the following table:

The online version of the original article can be found at http://dx.doi.org/ 10.1007/s11367-012-0455-2.

A. Pires $(\triangle) \cdot G$. Martinho

Departamento de Ciências e Engenharia do Ambiente, Faculdade de

Ciências e Tecnologia, Universidade Nova de Lisboa,

Caparica 2829-516, Portugal

e-mail: alp11931@fct.unl.pt 
For $1,000 \mathrm{~kg}$ of treated WLO Input

Rotary kiln emissions

(for ECR, ECER,

and CER scenarios)

Re-refined base oil

from re-refining

(R scenarios)

Electric energy from WLO recycling and energy

recovery (REP scenarios)

Steam

Output
Expanded clay (ECR scenarios)
Heat energy (for ECER and CER scenarios)
Carbon dioxide
Sulfur dioxide
Arsenic
Cadmium
Chromium
Nickel
Nitrogen oxides
Carbon monoxide
NMVOC

Sodium hydroxide

Propane

Hydrogen

Heat energy

Electric energy

Base oil

Flux oil

Light ends

Asphaltic residue

Sewage

Electric energy

\section{Electric energy}

Oiled water

Heavy residues

Carbon dioxide

Carbon monoxide

Hydrogen fluoride

Hydrogen sulfide

Cadmium

Chromium

Nickel

Nitrogen oxides

Sulfur dioxide

Particles
References

EIPPCB (2007), EMEP/

CORINAIR (2007),

EMEP/EEA (2009)

$3,109.5 \mathrm{~kg}$

$1.8 \mathrm{E}-1 \mathrm{~kg}$

$2.0 \mathrm{E}-8 \mathrm{~kg}$

$1.3 \mathrm{E}-6 \mathrm{~kg}$

$1.6 \mathrm{E}-8 \mathrm{~kg}$

$4.3 \mathrm{E}-6 \mathrm{~kg}$

$5.1 \mathrm{~kg}$

$16.1 \mathrm{~kg}$

$5.0 \mathrm{~kg}$

$0.7 \mathrm{~kg}$

$2.2 \mathrm{~kg}$

$2.0 \mathrm{~kg}$

$1.7 \mathrm{E}-1 \mathrm{MWh}$

6.7E-1 MWh

$7.8 \mathrm{E}-2 \mathrm{MWh}$

$725.2 \mathrm{~kg}$

$82.2 \mathrm{~kg}$

$14.2 \mathrm{~kg}$

$5.18 \mathrm{~kg}$

$79 \mathrm{~kg}$

3.6E-2 MWh APA (2008),

$3.2 \mathrm{MWh}$

EIPPCB (2006)

$46.7 \mathrm{~kg}$

$24.8 \mathrm{~kg}$

$2,078.5 \mathrm{~kg}$

$5.3 \mathrm{~kg}$

$4.4 \mathrm{E}-5 \mathrm{~kg}$

$3.4 \mathrm{E}-8 \mathrm{~kg}$

$9.7 \mathrm{E}-9 \mathrm{~kg}$

$4.8 \mathrm{E}-9 \mathrm{~kg}$

$3.9 \mathrm{E}-7 \mathrm{~kg}$

$23.6 \mathrm{~kg}$

$3.9 \mathrm{~kg}$

$1.29 \mathrm{~kg}$

ifeu (2005)

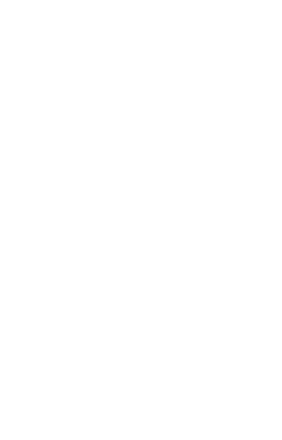

